\title{
Determination of Base Station Emission Power Change in a Mobile Network Cell with Movable Users
}

\author{
Aleksandar LEBL ${ }^{1}$, Dragan MITIC ${ }^{1}$, Branimir TRENKIC ${ }^{2}$, Zarko MARKOV ${ }^{1}$ \\ ${ }^{1}$ IRITEL AD Beograd, Batajnički put 23, 11080 Belgrade, Serbia \\ ${ }^{2}$ School of Electrical and Computer Engineering of Applied Sciences, Vojvode Stepe 283, 11000 Belgrade, Serbia
}

\{lebl, mita, zmarkov\}@iritel.com, btrenkic@viser.edu.rs

Submitted January 10, 2018 / Accepted July 31, 2018

\begin{abstract}
This paper considers base transceiver station (BTS) emission power change in the cell-organized mobile network with dynamic power control, due to users' moving. Such power adjustment contributes to energy saving and environment pollution reduction. We analyzed mutual influence of user's moving speed, users' surface distribution and propagation coefficient $\gamma$ on BTS power variations. It is proved that users' concentration near BTS, greater $\gamma$ (in urban areas), faster user's moving and greater connection duration contribute to BTS power increase of several tens of percent in real conditions. We present two examples when mean user's moving distance is $30 \%$ of mobile cell radius: 1) power of one movable user is increased more than 50\% when distance between user and BTS is uniformly distributed (decreasing users' surface density); 2) emission power is decreased 2.5\% when users are uniformly distributed (there are more users near a cell rim). BTS power has nearly constant value in the second example, because in our model users, crossing the cell rim, are replaced by users from adjacent cells, who are moving towards BTS. The analysis results are verified by Monte Carlo simulation, where user's starting position, displacement and angle of moving are determined based on randomly generated numbers.
\end{abstract}

\section{Keywords}

Base station, emission power, user's moving, handover

\section{Introduction}

Modern mobile communications are based on a dynamic management of the emission power from BTSs to users in order to reduce necessary power consumption. Emission power of the BTS cell towards the individual users is calculated, or the total emission power is determined in a number of papers [1-4]. In some papers influence of environment attenuation on the emission power is considered [5] and in the other ones influence of users' area distribution on the total BTS emission power is ana- lyzed [6]. It is supposed in all these papers that users are not moving during the connection duration. In this paper we consider how mobile users' moving in a cell and in a network affects the emission power towards such users, and the total emission power. The results from this paper show that BTS power may be dynamically adjusted during user's moving (UM), thus contributing to the program of energy saving in globally resource-optimized and energyefficient networks (GREEN).

Section 2 of this paper gives short presentation of existing models of UM in the area of BTS cell. After that, in Sec. 3 the assumptions and designations are introduced and a cell model is defined. Sections 4 and 5 suggest a method for calculation of emission power change due to users, who are moving in the cell. Estimation of emission power change, caused by users who need handover, is presented in Sec. 6. Section 7 deals with BTS power change as a result of UM for different users' density distributions in the BTS cells. In Sec. 8 we study the influence of the interference variations, caused by UM, on the performance of mobile user receiver. Simulation program and some of its results are given is Sec. 9. At the end, Section 10 is paper conclusion.

\section{Existing Models of User's Moving}

There are a significant number of papers which analyze different models of mobile UM in a BTS cell. Papers [7-11] are only a part of this literature. Authors in [7] are focused on determination of user's movement rules in such a way to keep the desired users' distribution in the cell (usually the uniform one). Two approaches are considered in order to achieve this goal: temporal random waypoint (RWP) selection and spatial RWP selection. In the first one user's speed is adjusted and changed during movement to keep the uniform users' distribution. This assumption does not correspond to real situation of more constant UM speed. The second approach is based on an inconvenient spatial selection of user, who moves, which leads to nearly unchanged, uniform users' distribution after movement, while exploiting real situation of constant user's speed 
during moving. Both approaches do not take into account real situation of a great number of cells in a mobile network and the process of handover (replacing the user control by a considered BTS on a cell rim to the control of adjacent BTS).

Opposite to [7], reference [8] is devoted to systems with moving of users within a number of cells (microcells), organized in groups covering area of a macrocell. The goal in this paper was to determine users' distribution among cells (based on transition matrices characterizing user random walk between cells), not their position in a single cell. The analysis, dealing with parameters of a single cell, should follow this contribution.

Authors in [9] present random direction model with location dependent parameterization (RD LDP). RD models, besides RWP models (as the one in [7]) are the most often used models in the analysis. Absolute movement (walk) angle, length, speed and movement time are random variables with uniform distribution, and the model specificity is that these random variables are defined as a function of starting point of next segment walk. Model definition in [9] allows real movement traces to be connected with simulation model, but the suggested model does not include user's behavior at the cell rim.

In real urban situations RD models may not be implemented, because directions of movement are influenced by straightforward, rectangular street structure. That's why mobile networks of Manhattan type are introduced [10]. Contribution of [10] is very detailed system simulation, which is based on traffic analysis according to Erlang queueing model. User's moving behavior is separately modelled for pedestrians and drivers. The analysis includes traffic loss and probability of successful handover. Traffic loss due to handover loss and due to lack of idle channels for new calls is related to the number of reserved channels for handover and to the number of channels devoted to newly generated traffic. But, BTS emission power is not included in this, detailed analysis.

Contribution [11] is a survey of different models for UM in a BTS cell. Besides already presented models in this section (which are most frequently implemented), some other models are also mentioned in [11]. First, we have Gauss-Markov mobility model and Smooth random mobility model, which allow that user's speed variations are presented in a more realistic way, than by random number generation. Then, mobility models with spatial dependence consider correlation of various UM (for example, vehicles moving one after the other on a highway, the groups of people who are working together, and so on).

It may be concluded from this presentation that there is a number of models, which try to present mobile users' moving as realistically as possible. However, no one of these papers analyzes how UM affect the change of BTS emission power.

There are a number of factors that have influence on the BTS power. The influence of intra-cell traffic value (intra-cell traffic corresponds to the situation when both users - participants in one connection are located in the same BTS cell area) is analyzed in [12]. Distance between an active user and BTS determines the BTS power devoted to the considered user. Related to this, users' surface density distribution has also influence on the BTS power [6], [13]. Besides, environment characteristics cause variation in signal attenuation on its way between the BTS and the mobile user. That's why it is necessary to adjust BTS emission power to transmit optimal signal power level to the user. As a short conclusion, it may be emphasized that there are three groups of factors, which have influence on the BTS power: characteristics of traffic, user's placement in the cell and signal propagation conditions. These factors may not be always analyzed completely separately; they are related one to the other. In this paper we focus on the analysis of UM influence (as an element in the second group of factors) on the BTS power. User's moving is considered together with two other groups of emphasized factors. The analysis in this paper is based on our contributions in [6], [12] and [13].

Besides the analysis of BTS power change as a consequence of UM, the other important element for the analysis is the variation of interference, also as a consequence of UM. In this paper we focus on the overview of this problem (we considered only the influence of users, moving in the same mobile cell, on the interference), giving basic guidelines for the future analysis.

\section{Cell Model, Designations and Assumptions}

Let us consider one cell of mobile network, Fig. 1.

A cell is circular, with radius $R$. The power of a signal, which is transmitted from a BTS to a mobile user (MS) at the distance $r$, has to be increased due to transmission attenuation according to the known expression $P=a \cdot r^{\gamma}$. In this expression $a$ is the coefficient of proportionality and $\gamma$ is the propagation coefficient $(\gamma=2-5$ [6], [14]). The number of channels, i.e. the maximum number of active users in one moment (when there is no intra-cell traffic) is $N$. The BTS sends signal power proportional to the mutual distance MS-BTS to each active user, i.e. to mobile station. That's why all active users receive the same signal power intensity. This method is called power control. In this paper we suppose that BTS power is changed continually, proportionally to the requirement, although, practically, power control is usually realized in steps of $2 \mathrm{~dB}$.

There are two types of UM: in the BTS cell and between cells. Let us consider moving in the cell of one user, who is in the beginning of a connection (which has mean duration $t_{\mathrm{m}}$ ) at a distance $r$ from a BTS, and his primary position is designated as MSp in Fig. 1(a). Variable $r$ is a random variable. During a connection time the user changes his position and in one moment is located at a distance $d$ from his primary position. His distance (MSm) 


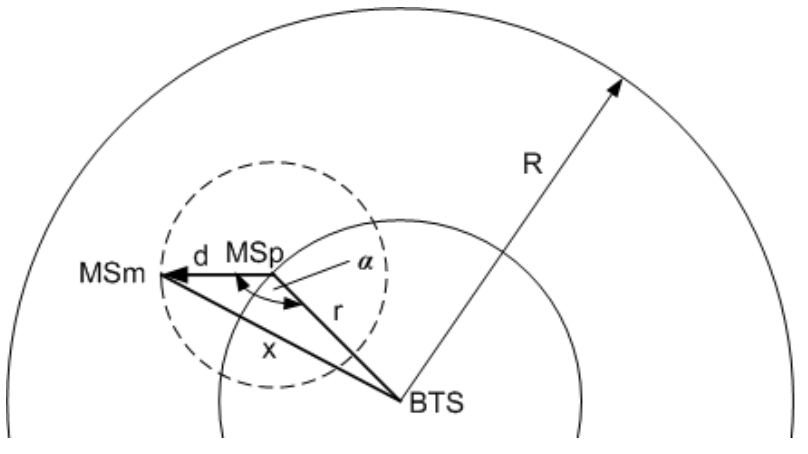

(a)

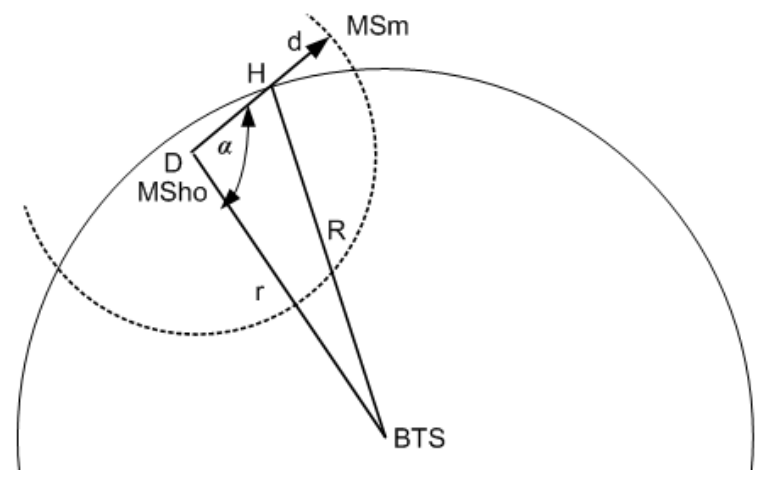

(b)

Fig. 1. User's moving in the BTS cell (a) and when handover is necessary (b).

\begin{tabular}{|c|c|c|}
\hline $\boldsymbol{r}$ and $\boldsymbol{d}$ & $\mathbf{\% O}$ & $\mathbf{\% I}$ \\
\hline$r=10 \cdot d$ & 51.6 & 48.4 \\
\hline$r=5 \cdot d$ & 53.19 & 46.81 \\
\hline$r=3 \cdot d$ & 55.35 & 44.65 \\
\hline$r=2 \cdot d$ & 58.05 & 41.95 \\
\hline$r=d$ & 66.7 & 33.3 \\
\hline$r=0.8 \cdot d$ & 71.5 & 28.5 \\
\hline$r=0.6 \cdot d$ & 81.36 & 18.4 \\
\hline$r=0.5 \cdot d$ & 100 & 0 \\
\hline
\end{tabular}

Tab. 1. Parts of the circle which increase $(\% O)$ and decrease (\%I) emission power when user is moving.

from BTS is now $x, x<R$. Variable $d$ is also a random variable. As is, in principle, $x \neq r$, it is obvious that MS moving causes variation of emission power transmitted to the considered MS. It is supposed that MS moving is possible and equally probable in all directions, i.e. that an angle $\alpha$ (Fig. 1(a)) is a random variable with uniform distribution. It means that user at distance $d$ from his primary position may be located at a circle with radius $d$ around primary position, MSp. An angle between the radius, which connects BTS and MSp and the radius $d$, which connects MSp and MSm, is designated by $\alpha$.

In the paper, the mean value of any random variable $x$ will be designated by an subscript ${ }_{m}$, i.e., for example, the mean value of $x$ is $x_{\mathrm{m}}$.

Moving through different cells, i.e. moving in a case of handover is specific. It is moving from one cell to the other ones [10], [15]. It is assumed that the mean number of users who cross from the considered cell to the adjacent ones is equal to the mean number of users who cross from the adjacent cells to the considered one. Let us choose one of such users, MSho, Fig. 1(b). When moving, this user starts from his primary position, $D$, crosses the cell rim, position $H$, in order to continue his moving in the neighbor cell and finishes moving at a distance $d$ from the starting position. It is assumed that the properties of adjacent cells are identical to that of the considered one.

\section{User's Moving in the Cell when there is no Handover}

The new position MSm is on the distance $d$ from the primary position, but it may be at any point of the circle with radius $d$ (dashed line), circumscribed around MS, as presented in Fig. 1(a). It is obvious from Fig. 1(a) that the circle with radius $d$ has greater arc outside the circle with radius $r$ than inside this circle with radius $r$. Table 1 presents parts of the circle, which are outside (O) or inside (I) the circle with radius $r$, depending on the relationship between $r$ and $d$.

All moving directions are equally probable. That's why it is more probable that user's distance from BTS increases over the primary distance $r$ ( $x$, MSm-BTS), than that this distance decreases under $r$, i.e.:

$$
P(x>r)>P(x<r) \text {. }
$$

Required emission power to the user in the primary position MS depends on the distance $r$ and necessary emission power to the user on the new position MSm depends on the distance $x$. That's why it may be concluded that, in principle, signal of greater power must be sent to the movable users than to the users, who are not moving.

\section{Determination of Mean Distance of Moving User within a Cell}

Distance of new user's position MSp from BTS is $x$, Fig. 2, and may be expressed as:

$$
x=\sqrt{r^{2}+d^{2}-2 \cdot r \cdot d \cdot \cos \alpha} .
$$

User's movement is completely random and there are three variables, which determine this randomness.

The first variable is the user's primary distance $r$ from the BTS. Its value may be in the range $0<r<R$. This value in random traffic process depends also on the area users' density distribution in the cell.

The second variable is the user's distance from the primary position for the connection duration, $d$. This variable depends on user's speed during the connection and on the connection duration.

The third variable is the angle $\alpha$ between the UM direction during the connection and the direction of the line between the user's primary position and the BTS. The value 


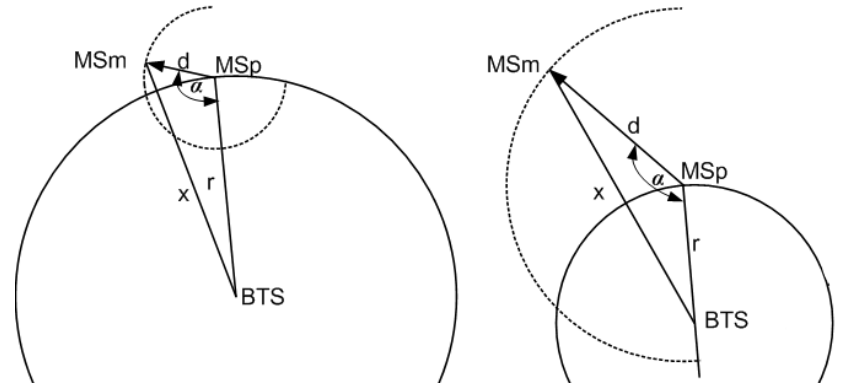

Fig. 2. New position determination when user is moving in a cell.

of angle $\alpha$ may be between $0^{\circ}$ and $360^{\circ}$, but $x$ takes all possible values from $|r-d|$ to $r+d$ if angle $\alpha$ is changed only from $0^{\circ}$ to $180^{\circ}$. That's why this range is considered, Fig. 2.

It is obvious that the new user's distance from BTS, $x$, is dependent random variable (random function), which depends on three independent random variables

$$
x=g(r, d, \alpha)=\sqrt{r^{2}+d^{2}-2 \cdot r \cdot d \cdot \cos \alpha} .
$$

Determination of the general expression for the new user's distance from the BTS may be complicate in some of the cases analyzed in this paper, [16]. Determination of random variable mean value in this case includes triple integral calculation with the possibility of users' density changing in the cell. That's why in such cases the distance is determined using calculation of the value $x$ for specific numeric values of $r, d$, and $\alpha$, as in ${ }^{1}$ and verified relatively simply using simulation procedures [17].

\section{User's Moving in the Case of Handover}

Let us consider user A, who moves from his primary position MSp towards the other cell and arrives in a new position NP1, Fig. 3. In order to determine necessary power for such a user, it is supposed that there is a user B from the adjacent cell, who passes symmetric way towards the considered cell (dashed line in Fig. 3). The way of this user finishes in the new position point NP2. In this movement the considered BTS loses jurisdiction of sending signal to the user A and accepts jurisdiction of sending signal to the user B.

Distance, $x$, of the user B position NP2 from BTS after handover may be determined by (3) and Fig. 3:

$$
\begin{aligned}
& x h=g(r, d, \alpha)=\sqrt{r^{2}+d^{2}-2 \cdot r \cdot d \cdot \cos \alpha}, \\
& x=2 \cdot R-x h .
\end{aligned}
$$

The distance of the arrived user B, $x$, Fig. 3, determines power, which is necessary to be delivered to this user. Significant number of users after handover approach

\footnotetext{
${ }^{1} \mathrm{http}: / /$ calculator.tutorvista.com/triple-integral-calculator.html
}

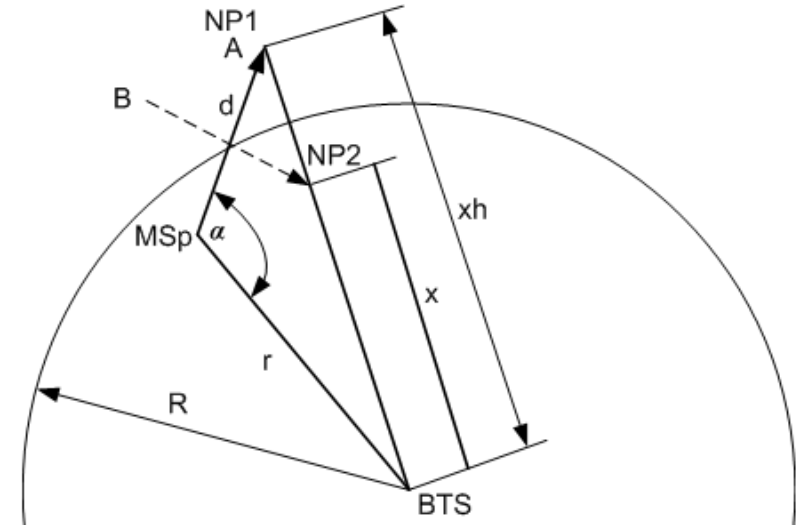

Fig. 3. New position determination in the case of handover.

BTS. That's why it may be expected that users after handover require less power than in the primary position.

\section{Necessary Power Relation for Stationary and Movable User}

The relation of mean emission power, which is necessary for a movable user, $P_{\mathrm{mx}}$, and mean emission power $P_{\mathrm{m}}$, necessary in primary position, will be designated as $A_{\gamma}$. It depends on the relation of the mean movable user's distance from the BTS, $x_{\mathrm{m}}$, and the primary distance from the BTS, $r_{\mathrm{m}}$. Relation of these two power values depends also on signal propagation coefficient, $\gamma$.

$$
A_{\gamma}=\frac{P_{\mathrm{mx}}}{P_{\mathrm{m}}}=\frac{a \cdot x_{\mathrm{m}}^{\gamma}}{a \cdot r_{\mathrm{m}}{ }^{\gamma}}=\left(\frac{x_{\mathrm{m}}}{r_{\mathrm{m}}}\right)^{\gamma} .
$$

It can be concluded that moving of a user in the cell increases the mean value of current distance and, as a consequence, necessary emission power. It is, also, possible that moving of a user after handover decreases the mean value of user's current distance from a BTS and that, so, necessary emission power for a movable user decreases. This means that influence of movable users on the BTS emission power depends on the users' density distribution in the cell. If users are concentrated around BTS, moving in the cell and, thus, power increase will predominate. On the contrary, if there are a lot of users near the cell rim, we may expect significant moving between cells, i.e. significant handover. In such a situation $x_{\mathrm{m}}$ decreases in relation to $r_{\mathrm{m}}$ and emission power decreases.

In order to prove this conclusion, we performed traffic process calculation and simulation in two cells with different users' density distributions. In the first cell users' density distribution is decreasing function from the BTS towards the cell rim. More precisely, distribution of user's primary distance from BTS, $r$, is uniform, i.e. user's distance as a random variable may have all values between 0 and $R$ with equal probability. In this case the mean value of user's distance from the BTS is $r_{\mathrm{m}}=0.5 \cdot R$. Such a cell is hereinafter referred to as a cell with decreasing users' density (CD). 
In the second cell surface users' density in the whole cell is uniform. It means that there are more users near the cell rim than near the BTS. In this second case the mean user's distance from the BTS is $r_{\mathrm{m}}=(2 / 3) \cdot R$, [5]. In the following text such a cell is referred to as the cell with uniform users' density (CU).

Both in $\mathrm{CD}$ and in $\mathrm{CU}$ cell, the choice of a movable user and his movement are random. The choice of the movable user is realized in accordance to the users' distribution in the cell. User's moving is equally probable if considering distance of movement (in a certain range) and direction (angle) of movement.

As already emphasized, calculation of mean relative value of new user's position distance from BTS, $x_{\mathrm{m}} / r_{\mathrm{m}}$, is complicated in the case of arbitrary users' distance distribution. But, in the case of CD users' distribution (i.e. when distribution of users is uniform as the function of user's distance from the BTS), the solution may be obtained solving the integral

$$
\begin{aligned}
& \frac{x_{\mathrm{m}}}{r_{\mathrm{m}}}= \\
& \frac{\int_{r=0}^{r_{\max }}\left(\int_{d=0}^{d_{\max }}\left(\int_{\alpha=0}^{\pi} \sqrt{r^{2}+d^{2}-2 \cdot r \cdot d \cdot \cos \alpha} \cdot \mathrm{d} \alpha\right) \cdot \mathrm{d} d\right) \cdot \mathrm{d} r}{r_{\max } \cdot d_{\max } \cdot \pi \cdot r_{\mathrm{m}}}
\end{aligned}
$$

where $r_{\max }$ is the maximum starting point distance of the user from the BTS and $d_{\max }$ is the maximum distance of UM.

The results of calculation for $\mathrm{CD}$ cell are presented in Fig. 4, where $x_{\mathrm{m}} / r_{\mathrm{m}}$ is presented as a function of the mean value of movable user's displacement, $d_{\mathrm{m}} / R$. It is clear, according to Fig. 4, that the mean value of movable (in the cell) user's distance from the BTS is always greater than the user's primary distance from BTS, $x_{\mathrm{m}}>r_{\mathrm{m}}$. Distribution of the primary user's distance from the BTS, $r$, is uniform in the range $(0, R)$. User's displacement depends on user's speed. Its distribution during the connection is always uniform and may be considered as a random variable in six ranges:

in the range $(0,0.1 R)$ where the mean value is $d_{\mathrm{m}} / R=0.05$, in the range $(0,0.2 R)$ where the mean value is $d_{\mathrm{m}} / R=0.1$, in the range $(0,0.3 R)$ where the mean value is $d_{\mathrm{m}} / R=0.15$, in the range $(0,0.4 R)$ where the mean value is $d_{\mathrm{m}} / R=0.2$, in the range $(0,0.5 R)$ where the mean value is $d_{\mathrm{m}} / R=0.25$, in the range $(0,0.6 R)$ where the mean value is $d_{\mathrm{m}} / R=0.3$.

Verification of these results shows that results of calculation and simulation agree very well.

Figure 5 presents relative increase of the mean necessary emission power as a function of coefficient $\gamma$ and the mean value of movable user's displacement, $d_{\mathrm{m}} / R$ if users' density is decreasing, i.e. if $\mathrm{CD}$ cell is considered. For users, who move fast, necessary power increase may be even several tens of percent.

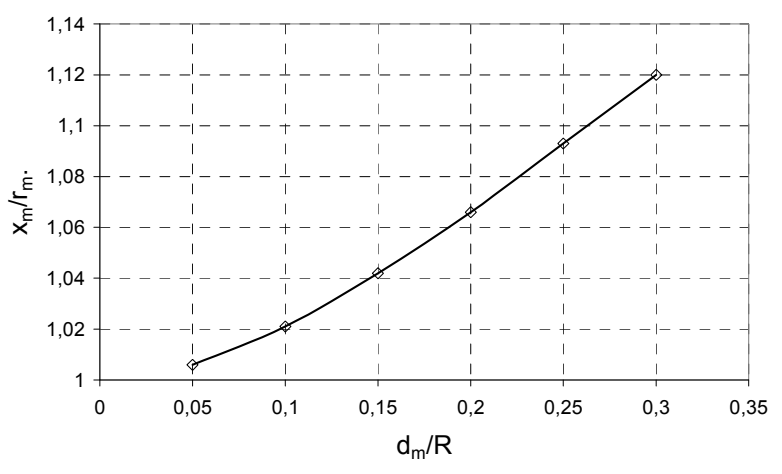

Fig. 4. Relative mean value of the new user's position distance, $x_{\mathrm{m}} / r_{\mathrm{m}}$, as a function of the mean movable user displacement, $d_{\mathrm{m}} / R, \mathrm{CD}$ cell $r_{\mathrm{m}}=0.5 \cdot R$.

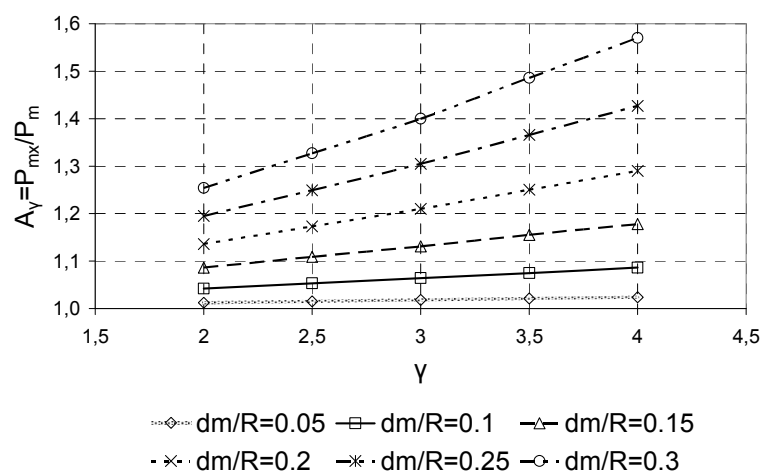

Fig. 5. Relative increase of the mean necessary emission power for one user $A_{\gamma}$, as a function of $\gamma$ and the mean displacement of movable user, $d_{\mathrm{m}} / R$ at $r_{\mathrm{m}}=0.5 \cdot R$ in CD cell.

Calculation of $\mathrm{CU}$ cell is realized in the same way as for $\mathrm{CD}$ cell: we consider six ranges of UM, depending on user's speed. Surface users' density is uniform in the whole cell, so there are a lot of users near the cell rim. That's why probability of UM towards adjacent cells is significant. We suppose that neighbor cells have similar characteristics as the considered one, so there is significant probability of movable users arriving from these neighbor cells.

Figure 6 presents the relative mean value of the new user's position distance from the BTS, $x_{\mathrm{m}} / r_{\mathrm{m}}$, as a function of the mean value of a movable user's displacement, $d_{\mathrm{m}} / R$,

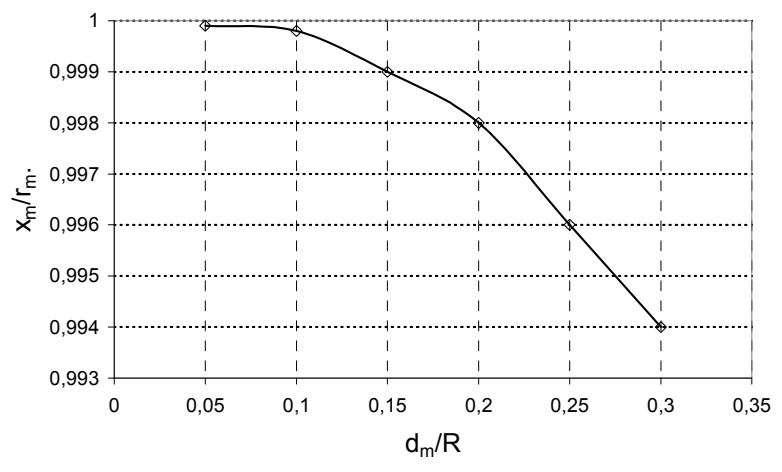

Fig. 6. Relative mean new user's position distance, $x_{\mathrm{m}} / r_{\mathrm{m}}$, as a function of the mean displacement of a movable user, $d_{\mathrm{m}} / R$ at $r_{\mathrm{m}}=2 \cdot R / 3$ in CU cell. 


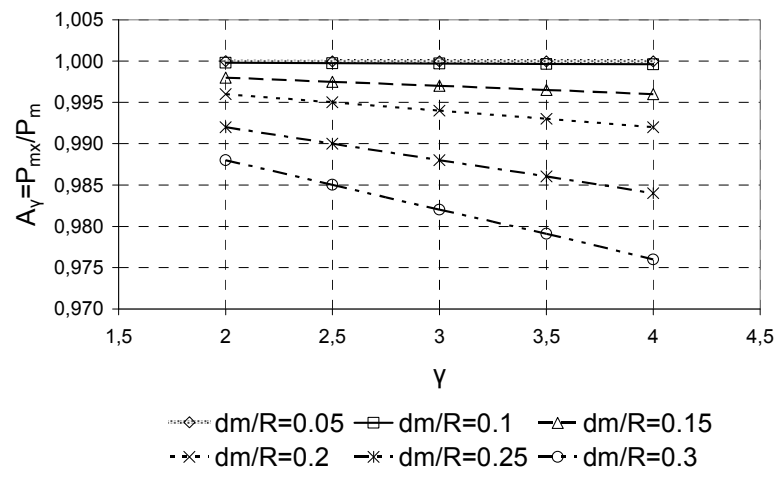

Fig. 7. Relative decrease of the mean necessary emission power for one user $A_{\gamma}$, as a function of $\gamma$ and the mean displacement of movable user, $d_{\mathrm{m}} / R$ at $r_{\mathrm{m}}=2 \cdot R / 3$ in CU cell.

in CU cell. It is obvious that the mean value of the movable user's distance from the BTS decreases. This can be attributed to the impact of a greater number of handovers.

Figure 7 presents the relative change of the mean necessary emission power of one movable user $A_{\gamma}$, as a function of coefficient $\gamma$ and the mean value of movable user's displacement, $d_{\mathrm{m}} / R$, for $\mathrm{CU}$ cell. It is obvious that power for movable users decreases, but in a very small extent. Even in a case of fastest $\mathrm{UM}\left(d_{\mathrm{m}} / R=0.3\right)$ power decrease is not greater than several percent.

The change of BTS power is the greatest in the time of the greatest traffic load and it may be easily estimated as a product of the mean power change for one user (Fig. 5 and Fig. 7) and the mean number of active users, who are moving during a connection.

The model of UM, which we developed, may be classified as random direction (RD) model. The main results of this paper are related to BTS power change as the result of UM and are presented in Fig. 5 and Fig. 7. We included the real situation of users' handover existence between BTSs at the cell rim and the influence of environment physical characteristics (propagation coefficient) in the analysis. According to our knowledge, there is no similar analysis dealing with RD models ([9], [18]), where BTS emission power is considered. That's why we couldn't compare our main results with the results from some other contribution.

RWP models ([7]) of UM are developed with the aim to maintain the same user distribution after moving of users. Indirectly, BTS power in such models remains the same after users' moving. This assumption is appealing for consideration ([18]), but, the model does not completely reflect the real situation in practice that BTS power may be changed when users are moving.

Example. Let us consider two cells: the first one with uniform users' density (CU) and the second one with decreasing users' density (CD). The radius of both cells is $R=5 \mathrm{~km}$ and $20 \%$ of users in both cells are moving during connection at the speed $v=60 \mathrm{~km} / \mathrm{h}$. The mean connection duration is $t_{\mathrm{m}}=2 \mathrm{~min}$. Propagation coefficient is $\gamma=3.5$. The mean value of displacement during a connection is $d_{\mathrm{m}}=0.5 \cdot v \cdot t_{\mathrm{m}}=1 \mathrm{~km}$. It means that $d_{\mathrm{m}} / R=0.2$.
For the adopted values in the case of CD cell, it may be found in Fig. 5 that power change for movable users is $A_{3.5} \approx 1.25$. BTS power increase is about $5 \%$, as $20 \%$ of users are moving.

In the case of CU cell, it may be found in Fig. 7 that power change for movable users is $A_{3.5} \approx 0.993$. BTS power decrease is negligible, i.e. $0.014 \%$.

\section{Influence of Interference}

Performance of mobile user's receiver is expressed by the ratio of the part of BTS power intended for the considered user to the power devoted to all other active users in the system. The power intended to all active users except the considered user represents interference. According to [19], when considering the total interference, it is necessary to take into account active users from the same cell, where the analyzed user moves, and also the users from the surrounding cells. The number of interfering rings depends on the value of $\gamma$. When $\gamma$ decreases, it is necessary to calculate influence of the users from the greater number of interfering rings.

In our following brief analysis we consider a simplified model, where only interference caused by the active users from the same cell is supposed. According to (1) from [19], interference received by the considered user, who is at the distance $r$ from the BTS, may be, approximately, expressed as:

$$
I=\alpha \cdot P \cdot r^{-\gamma}
$$

where $P$ is the total BTS emission power and $\alpha$ is the constant of proportionality.

Let us, further, suppose that the BTS power increases with the constant of proportionality $k$, as a consequence of UM, i.e.

$$
P_{\mathrm{mov}}=k \cdot P \text {. }
$$

The interference after UM will be:

$$
I_{\mathrm{mov}}=\alpha \cdot k \cdot P \cdot x^{-\gamma}
$$

The power for the considered user is adjusted according to his distance from the BTS. It means that the receiver always receives approximately the same power level intended for its function, regardless of the user's distance from the BTS. That's why it is necessary that interference is also the same after UM to keep the same receiver performance. If we equate interference before UM and after moving, on the base of (7) and (9) we obtain that relation of user's distance from the BTS before moving $(r)$ and after moving $(x)$ is:

$$
\frac{x}{r}=k^{\left(\frac{1}{\gamma}\right)} .
$$

It means that, although the BTS power is increased as a consequence of UM, interference may be decreased. 
Interference will be decreased for the users, who run away from the BTS in such a way that their distance after moving is greater than value $x$ calculated by (10). On the contrary, users, who are moving towards the BTS, may request greater power level due to interference.

The presentation in this section does not consider interference caused by active movable users from surrounding rings. Such, complete, analysis will be the subject of future calculation and simulation.

\section{Verification}

Verification is based on traffic process simulation in the network, [17], [20]. This simulation program is our original development, realized in $\mathrm{C}++$ programming language. It is the extended version of simulation programs, implemented for the analysis in [6], [12] and [13]. The purpose of extension is to model UM in the BTS cell area. User's moving is modelled in a very detailed fashion: for each connection separately the user's starting location (distance from the BTS), the direction of UM (angle in relation to the cell radius) and the distance of movement is determined. Environment influence, expressed by propagation coefficient $\gamma$, is included in the simulation process. But, in this moment simulation program does not include possibility to determine influence of interference variation (as a consequence of UM) on the BTS power. In our further analysis we are going to improve program performances including this element.

A simulation method to some extent depends on the performed analysis (whether handover is considered or not, i.e. whether UM is random in the sense of its length and angle, or these are constant, average, values, what characteristic is determined as the final result (power or distance), and so on). Flow-chart in Fig. 8 corresponds to one of simulated cases (implemented handover and random distance and angle of UM). The parts of the simulation program, which are related to UM and which are new comparing to the previous program versions (from [6], [12] and [13]) are highlighted grey in Fig. 8.

Meaning of the designations in Fig. 8 is: $r$ - initial distance BTS-MS, $d$ - distance of UM, $\alpha$ - angle of UM in relation to the line, which connects BTS and MS, $x$ - distance BTS-MS after movement completion, step - current number of realized connections.

Program execution starts with initialization in block 1 . After initialization of variables in block 1 , the first step in simulation procedure is a new call generation on the base of random number RN0 generation, according to classical Monte Carlo or roulette telephone traffic simulation (block 2). We have taken over this part of simulation program from literature (for example [21]) and implemented it in the same way as in [6], [12], [13] and [17].

Initial distance BTS-MS $(r)$ is determined in block 3 , on the base of random number RN1. It is our addition to

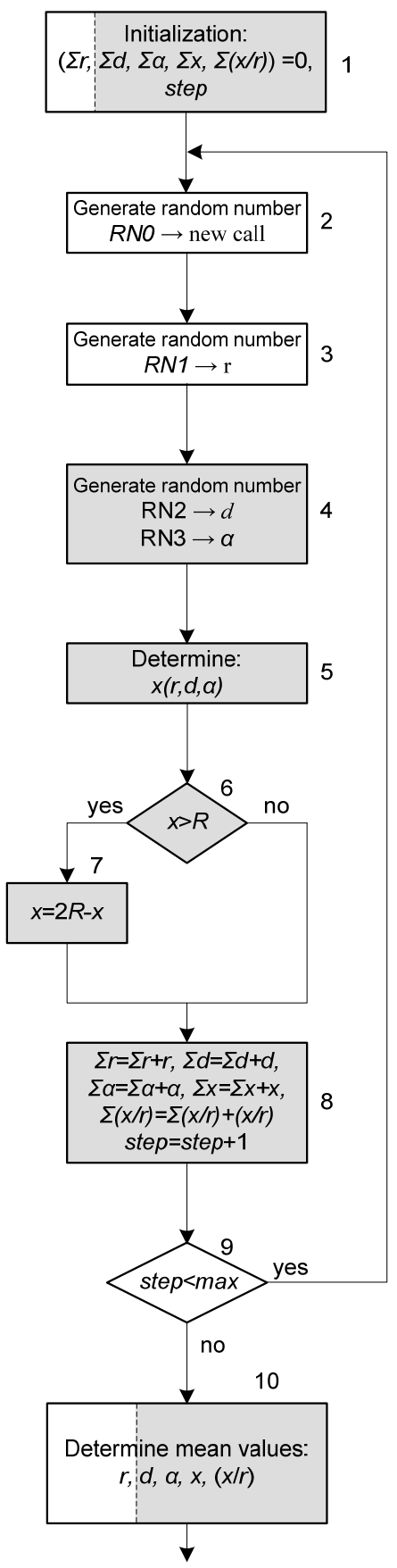

Fig. 8. The flow-chart of simulation program.

classical traffic simulation, intended for the determination of user's distance from the BS, which is already implemented and described in [6], [12], [13] and [17].

Besides $r$, two other variables, which define current MS position as the result of moving ( $d$ and $\alpha$ ) in the simulation are generated using random numbers (RN2, RN3) in block 4. After this, the value $x$ is determined according to (3) in block 5. If $x$ is greater than the BTS cell radius $(R)$, MS leaves the considered cell (it is not taken into account in further contributions to the results of simulation), i.e. handover is supposed. It means that MS is replaced by the corresponding MS, which arrived from the neighbor BTS. This new MS distance is corrected according to the expres- 


\begin{tabular}{|c|c|c|}
\hline Analysis conditions & $\boldsymbol{x}_{\mathrm{m}} / \boldsymbol{r}_{\mathrm{m}}$ simulation & $\boldsymbol{x}_{\mathrm{m}} / \boldsymbol{r}_{\mathrm{m}}$ calculation \\
\hline$r_{\mathrm{m}}=0.5 R, d_{\mathrm{m}}=0.05 R$ & 1.0058 & 1.0060 \\
\hline$r_{\mathrm{m}}=0.5 R, d_{\mathrm{m}}=0.10 R$ & 1.0213 & 1.0211 \\
\hline$r_{\mathrm{m}}=0.5 R, d_{\mathrm{m}}=0.15 R$ & 1.0418 & 1.0427 \\
\hline$r_{\mathrm{m}}=0.5 R, d_{\mathrm{m}}=0.20 R$ & 1.0668 & 1.0660 \\
\hline$r_{\mathrm{m}}=0.5 R, d_{\mathrm{m}}=0.25 R$ & 1.0950 & 1.0932 \\
\hline$r_{\mathrm{m}}=0.5 R, d_{\mathrm{m}}=0.30 R$ & 1.1200 & 1.1207 \\
\hline
\end{tabular}

Tab. 2. Mean movable user's distance in relation to primary distance, the results of simulation and calculation

sion in block 7. After that, the current total aggregate values of all the input and output values, mentioned in the description of the simulation, accumulate in block 8 .

Program execution completes by the determination of mean values of $r, d, \alpha, x$ and $x / r$ in block 10 .

Table 2 presents some results of simulation and calculation of the mean distance of a movable user from the BTS, $x_{\mathrm{m}}$, equation (2), for the specific case where the values are same as in Fig. 4, CD cell. Simulation and calculation conditions are presented in the first column of Tab. 2. The mean values of relative user's distance from the BTS, obtained by five simulation trials with 300000 realized connections each $\left(x_{\mathrm{m}} / r_{\mathrm{m}}\right.$ simulation), are presented in the second column of Tab. 2. The duration of each simulation trial with 300000 realized connections is about two minutes. The mean values of the relative user's distance from the BTS, obtained by calculation $\left(x_{\mathrm{m}} / r_{\mathrm{m}}\right.$ calculation $)$, are presented in the third column.

\section{Conclusion}

This paper considers the model of random direction (RD) of UM in which user's starting position, distance and angle of UM are random variables. Comparing to other contributions analyzing RD models (as, for example, [9], [18]), our paper estimates the value of emission power and percent of this power change in the cell with BTS power control, as a consequence of UM. Such power analysis is rare even in other models, which include UM. Comparing to some contributions, which tend to keep unchanged users' distribution during their moving, we allow variations in users' distribution, which is, in fact, proved in our paper to be real assumption. Moving in the cell increases necessary power and this increase is as greater as user's speed is greater, connection duration is longer, primary user's position is nearer to the BTS and propagation coefficient is greater. On the contrary, UM after handover decreases necessary emission power. This decrease is as greater as moving speed is greater, connection is longer and coefficient $\gamma$ is greater. The greatest influence on the change of necessary power has users' distribution in the cell. Power increase is necessary for movable users if their concentration is greatest in the center of the cell, i.e. around the BTS. The great users' concentration near the cell rim may cause power decrease for movable users. The total BTS emission power change is proportional to the part of users, who are moving.

\section{Acknowledgments}

This paper is realized in the framework of the projects TR32051 and TR32007, which are financed by the Ministry of Education, Science and Technological Development of the Republic of Serbia.

\section{References}

[1] MITIĆ, D., LEBL, A., MARKOV, Ž. Influence of traffic model on the calculation of BTS output power in GSM network. $A E \ddot{U}$ International Journal of Electronics and Communications, 2015, vol. 69, no. 5, p. 836-840. DOI: 10.1016/j.aeue.2015.02.003

[2] COLOMBI, D., THORS, B., PERSSON, T., et al. Downlink power distributions for $2 \mathrm{G}$ and $3 \mathrm{G}$ mobile communication networks. Radiation Protection Dosimetry, 2013, vol. 157, no. 4, p. 477-487. DOI: $10.1093 / \mathrm{rpd} / \mathrm{nct} 169$

[3] JOSHI, P., AGRAWAL, M., THORS, B., et al. Power level distributions of radio base station equipment and user devices in a $3 \mathrm{G}$ mobile communication network in India and the impact on assessments of realistic RF EMF exposure. IEEE Access, 2015, vol. 3, p.1051-1059. DOI: 10.1109/ACCESS.2015.2453056

[4] JOSHI, P., COLOMBI, D., THORS, B., et al. Output power levels of $4 \mathrm{G}$ user equipment and implications on realistic RF EMF exposure assessment. IEEE Access, 2017, vol. 5, p. 4545-4550. DOI: 10.1109/ACCESS.2017.2682422

[5] JOVANOVIĆ, P., MILEUSNIĆ, M., LEBL, A., et al. Calculation of the mean output power of base transceiver station in GSM. Automatika, 2014, vol. 55, no. 2, p. 182-187. DOI: 10.7305/automatika.2014.06.373

[6] MILEUSNIĆ, M., POPOVIĆ, M., LEBL, A., et al. Influence of users' density on the mean base station output power. Elektronika ir Elektrotechnika, 2014, vol. 20, no. 9, p. 74-79. DOI: 10.5755/j01.eee.20.9.5418

[7] MITSCHE, D., RESTA, G., SANTI, P. The random waypoint mobility model with uniform node spatial distribution. Wireless Networks, 2014, vol. 20, no. 5, p. 1053-1066. DOI: 10.1007/s11276-013-0661-2

[8] AKYLDIZ, I. F., LIN, Y.-B., LAI, W.-R., et al. A new random walk model for PCS networks. IEEE Journal on Selected Areas in Communications, 2000, vol. 18, no. 7, p. 1254-1260. DOI: $10.1109 / 49.857925$

[9] GLOSS, B., SCHARF, M., NEUBAUER, D. A more realistic random direction mobility model. In $4^{\text {th }}$ Management Committee Meeting. Würzburg (Germany), October 2005, p. 1-11.

[10] OLMOS, K., PIERRE, S., BOUDREAULT, Y. Traffic simulation in urban cellular networks of Manhattan type. Computer and Electrical Engineering, 2003, vol. 29, no. 3, p. 435-461. DOI: $10.1016 / \mathrm{S} 0045-7906(01) 00032-5$

[11] BAI, F., HELMY, A. A survey of mobility models in wireless adhoc networks. Chapter 1 in Wireless Ad Hoc Networks. Kluwer Academic, 2006, p. 1-30.

[12] MILEUSNiĆ, M., JOVANOVIĆ, P., POPOVIĆ, M., et al. Influence of intra-cell traffic on the output power of base station in GSM. Radioengineering, 2014, vol. 23, no. 2, p. 601-608. ISSN: 1805-9600 (online)

[13] LEBL, A., MITIĆ, D., POPOVIĆ, M., et al. Influence of mobile users' density distribution on the CDMA base station power. Journal of Electrical Engineering, 2016, vol. 67, no. 6, p. 390-398. DOI: $10.1515 /$ jee-2016-005 
[14] EBERSPRÄCHER, J., VÖGEL, H. J., BETTSTETTER, CH. GSM, Switching, Services and Protocols. (Chapter 5) $2^{\text {nd }}$ ed. John Wiley \& Sons, 2001. ISBN: 9780471499039. DOI: $10.1002 / 0470841745 . \operatorname{ch} 5$

[15] HONG, D., S. RAPPAPORT, S. Traffic model and performance analysis for cellular mobile radio telephone systems with prioritized and nonprioritized handoff procedures. IEEE Transactions on Vehicular Technology, 1986, vol. 35, no. 3, p. 77-92. DOI: 10.1109/T-VT.1986.24076

[16] PAPOULIS, A. Probability, Random Variables and Stochastic Processes. (Chapter 5) $3^{\text {rd }}$ ed. McGraw Hill, 1991. ISBN: 0-07048477-5

[17] MILEUSNIĆ, M., ŠUH, T., LEBL, A., et al. Use of computer simulation in estimation of GSM base station output power. Acta Polytechnica Hungarica, 2014, vol. 11, no. 6, p. 129-142. DOI: 10.12700/APH.11.06.2014.06.8

[18] NAIN, P., TOWSLEY, D., LIU, B., et al. Properties of random direction models. In Proceedings of the $24^{\text {th }}$ International Joint Conference of the IEEE Computer and Communications Societies (INFOCOM 2005). Miami (USA), 2005, p. 1897-1907. DOI: 10.1109/INFCOM.2005.1498468

[19] ISMAIL, M. S., RAHMAN, T. A. Forward-link performance of CDMA cellular system. IEEE Transactions on Vehicular Technology, 2000, vol. 49, no. 5, p. 1692-1696. DOI: $10.1109 / 25.892574$

[20] AKIMARU, H., KAWASHIMA, K. Teletraffic, Theory and Applications. $2^{\text {nd }}$ ed. Springer, 1999. DOI: 10.1007/978-1-44710871-9

[21] KOSTEN, L. Simulation in teletraffic theory. In The 6th International Teletraffic Congress ITC. Münich (Germany), 1970, p. 411-1-8.

\section{About the Authors ...}

Aleksandar LEBL was born in Zemun, Serbia, in 1957. He received his B.Sc. and M.Sc. from the Faculty of Electrical Engineering in Belgrade, Republic of Serbia, in 1981 and 1986, respectively, and his Ph.D. from the Faculty of Technical Science in Novi Sad, in 2009. He was employed from 1981 in the Switching Department of the Institute for Electronics and Telecommunications IRITEL in Belgrade and from 2015 he is with the Radio Communication Department, also in IRITEL. During years he worked on the project of Digital Switching System for Serbian Telecommunication Industry.

Dragan MITIĆ was born in Belgrade, Serbia, in 1953. He received his B.Sc. and M.Sc. from the Faculty of Electrical Engineering in Belgrade, Republic of Serbia, in 1977 and 1984, respectively, and his Ph.D. from the Faculty of Technical Science in Novi Sad, in 2002. Dr Mitić is a science advisor in IRITEL, Institute for Electronics and Telecommunications, Belgrade, Serbia. From 1977 until 1989 he was employed at the Land Forces Military Technical Institute in Belgrade, and since 1989 in IRITEL. He is author or co-author of more than 160 international and national scientific and professional papers. He works on several research projects for equipment of specific applications.

Branimir TRENKIĆ was born in Ćuprija, Serbia, in 1961. In 1987 he received his Graduate degree of Mathematics (Dipl-Math) at the University of Belgrade. He received the MSEE degree in Electronics and Communication Engineering Department from the University of Belgrade in 1997, and the Ph.D. degree in Electrical Engineering and Computer Sciences from the University of Novi Sad, in 1998. Dr. Trenkić joined the Faculty of Computer Sciences in Belgrade as associate professor in 2011. In 2013 he became a full professor at the Faculty of Computer Sciences and in 2016 he joined the School of Electrical and Computer Engineering of Applied Studies. Area of work: traffic modelling, performance analysis of communication networks and queueing strategies for communication networks.

Žarko MARKOV was born in Žitište, Serbia, in 1946. He received his B.Sc., M.Sc. and Ph.D. from the Faculty of Electrical Engineering in Belgrade, Republic of Serbia, in 1969, 1975, and 1976, respectively. Dr. Markov is a scientific counsellor in IRITEL, Institute for Electronics and Telecommunications, Belgrade, Serbia. Area of work: Switching technics, teletraffic theory, network signaling. $\mathrm{He}$ is author or co-author of more than hundred papers and six books. At the University of Belgrade, School of Electrical Engineering, Dr. Markov was a professor at the course of Switching Technics and Network Signaling. 